\title{
Clinical factors predicting drug-induced liver injury due to flucloxacillin
}

This article was published in the following Dove Press journal: Drug, Healthcare and Patient Safety

\section{Mikaela Lindh' \\ Pär Hallberg' \\ Qun-Ying Yue ${ }^{2}$ \\ Mia Wadelius'}

'Department of Medical Sciences, Clinical Pharmacology and Science for Life Laboratory, Uppsala University, Uppsala, Sweden; ${ }^{2}$ Swedish Medical Products Agency, Uppsala, Sweden
Correspondence: Mia Wadelius Department of Medical Sciences, Clinical Pharmacology and Science for Life Laboratory, Uppsala University, Uppsala University Hospital, Uppsala, SE-75I 85, Sweden

$\mathrm{Tel}+46$ I8 6II 4945

Fax +46 I 8552562

Email mia.wadelius@medsci.uu.se
Objectives: Drug-induced liver injury (DILI) is a serious adverse reaction due to flucloxacillin. The pathogenesis is not fully understood. Female sex, age over 60 years, and a longer treatment duration have been suggested to be predisposing factors. Carriers of HLA-B*57:01 have an 80 -fold increased risk, but due to the rarity of the reaction, testing of all patients is not cost-effective. We aimed to validate and detect clinical risk factors for flucloxacillin DILI. Methods: Clinical characteristics of flucloxacillin-treated patients with $(n=50)$ and without DILI $(n=2,330)$ were compared in a retrospective case control study. Cases were recruited from the Swedish database of spontaneously reported adverse drug reactions. Treated controls were selected from the Swedish Twin Registry. Statistical comparisons were made using chi-squared test and logistic regression. The significance threshold was set to $P<0.00357$ to correct for multiple comparisons. Reliable variables were tested in a multiple regression model.

Results: DILI was associated with female sex, OR 2.79, 95\% CI 1.50-5.17, $P=0.0011$, and with a history of kidney stones, OR 5.51, 95\% CI 2.21-13.72, $P=0.0003$. Cases were younger than controls, OR per increase in years $0.91,95 \%$ CI $0.88-0.94, P<0.0001$, probably due to selection bias. No difference in treatment duration was detected, OR 1.03, 95\% CI 0.98-1.08, $P=0.1790$.

Conclusion: We established female sex as a risk factor for flucloxacillin-induced DILI, and a history of kidney stones was identified as a potential risk factor. Clinical risk factors for flucloxacillin-induced DILI could be used to indicate whom to test for HLA-B*57:01 before treatment. Keywords: floxacillin, chemical and drug induced liver injury, drug-related side effects and adverse reactions, alanine transaminase, bilirubin

\section{Introduction}

Flucloxacillin is an orally administrated beta-lactam antibiotic that is frequently used in Europe, especially against Staphylococcus aureus infections of the skin and soft tissues. ${ }^{1,2}$ Drug-induced liver injury (DILI) is a collective term of different liver injuries as adverse drug reactions (ADRs) due to various substances. ${ }^{3}$ DILI is a known but unusual ADR due to flucloxacillin, and the prevalence among first-time users has been estimated to be $1 / 10,000 .{ }^{1}$ Most of the cases occur within 3 months of exposure to flucloxacillin. ${ }^{1}$ Flucloxacillin-induced DILI is a type B ADR, sometimes referred to as idiosyncratic. Such reactions are not related to the pharmacological effect or dose of the drug, ${ }^{4-6}$ and due to their rarity and unpredictability they are seldom detected in preclinical and clinical drug trials. ${ }^{7}$ Additionally, their rarity makes them difficult to investigate in epidemiological studies. ${ }^{8}$ DILI due to flucloxacillin is mostly cholestatic, ${ }^{1,2}$ but the pathogenesis of the injury is, as for 
type B ADRs and DILI in general, poorly understood. Both immune-mediated and metabolic hypotheses have been suggested. ${ }^{9}$ The immune-mediated hypothesis is based mainly on a T-cell-mediated and human leukocyte antigen (HLA) system associated response. ${ }^{10}$ According to the metabolic hypothesis, CYP3A4 derived metabolites of flucloxacillin can be toxic to biliary epithelial cells. ${ }^{11}$ Which hypothesis is true has not been proven, and it can be assumed that none is fully comprehensive.

A genome-wide association study (GWAS) has shown a significant association between flucloxacillin-induced DILI and the HLA-B*57:01 genotype. ${ }^{12}$ This HLA type is common in northern Europe, and estimated to occur in $6 \%-8 \%$ of the general European population. ${ }^{3,12}$ The GWAS showed that $84.3 \%$ of all patients with flucloxacillin-induced DILI carry HLA-B*57:01, and that carriers have an 80-fold risk of being affected, OR 80.6, 95\% CI 22.8-284.9. ${ }^{12}$ Although this is one of the strongest genetic associations with DILI, the high frequency of HLA-B*5701 in the white population coupled with the low incidence of DILI in treated patients gives it a low positive predictive value. ${ }^{12}$ This means that the majority of flucloxacillin-treated HLA-B*57:01 carriers will not develop DILI. ${ }^{13}$ It would be valuable to identify other predisposing factors for flucloxacillin-induced DILI that could help guide whom to test for HLA-B*57:01. ${ }^{14}$ Until now, female sex, age older than 60 years, and treatment duration longer than 2 weeks have been proposed as risk factors. ${ }^{1,2}$ This study aimed to validate previously suggested predisposing factors, and to identify new clinical risk factors for flucloxacillin-induced DILI.

\section{Materials and methods}

A retrospective case control study was performed using the SWEDEGENE database of clinical information on individuals affected by suspected ADRs (www.swedegene.se). SWEDEGENE is a collaboration between Uppsala University, the Swedish Medical Products Agency (MPA), and Karolinska Institutet, Sweden. Collaboration with the TWINGENE database at Karolinska Institutet allowed access to clinical information from twins born 1911-1958. ${ }^{15}$

\section{Ethics}

Ethical approval for the SWEDEGENE project was given by the regional ethics committee in Uppsala 2008-08-16 (Dnr 2008/213) and 2010-06-30 (Dnr 2010/231). Ethical approval for TWINGENE was given by the regional committee in Stockholm 2007-06-13 (Dnr 2007-644-31). Data were de-identified and study procedures were in accordance with the ethical standards of the regional ethics committees and the Declaration of Helsinki. All participants gave informed consent.

\section{Patient recruitment and data collection}

All cases with reports of suspected DILI between 1990 and 2016 were collected from the Swedish National database of spontaneously reported ADRs that is kept at the MPA. Every reporter was asked if the patient was suitable to be included in the study. Patients could also be referred from collaborating colleagues or contact SWEDEGENE directly. Each patient was then contacted, and if permission was granted, he or she was sent a study kit containing a questionnaire, an informed consent form, and a blood sample kit for DNA analysis (not applied in this study). The questionnaire included questions concerning, eg, sex, age, suspected causative drug(s), all concomitant drugs taken within 3 months before the ADR, diseases before the ADR, and more. The questionnaire was completed through a telephone interview. If additional information was required, copies of the participants' medical journals were requested. All information collected was then entered into the SWEDEGENE database.

\section{Inclusion and exclusion criteria}

All patients recruited by SWEDEGENE were required to be at least 18 years old and able to give informed consent. Inclusion and exclusion criteria were based on a definition of DILI as described in detail elsewhere (Tables 1 and 2). ${ }^{16-19}$ In brief, patients were excluded if they had been diagnosed with a liver or gallbladder condition since 1967 or if there was another more likely cause for the liver disorder, such as positive serology.

\section{Controls}

Treated controls were collected from the TWINGENE database. An excerpt from the Swedish National Drug Register containing all prescriptions dispensed at pharmacies from July 2005 until December 2014 was used to identify those with a prescription for flucloxacillin within this time. If an individual had collected several prescriptions for flucloxacillin, data around the first prescription were used in this study. The Swedish National Inpatient Register (IPR) was searched to find concomitant diagnoses using diagnosis codes according to the Swedish version of the WHO's tenth revision of the International Statistical Classification of Diseases and Related Health Problems, 10th revision, Swedish version (ICD-10-SE). ${ }^{20}$ The Swedish IPR contains diagnoses set within inpatient hospital care from 1964 and onwards. ${ }^{21}$ Since 2001, this register also contains hospital-based outpatient physician visits, but not primary health care data on a national 
Table I Inclusion criteria used for cases and reference values for liver function tests

\begin{tabular}{|c|c|}
\hline \multicolumn{2}{|c|}{ Inclusion criteria used for cases } \\
\hline \multicolumn{2}{|c|}{ I) At least one of following: ${ }^{a}$} \\
\hline \multicolumn{2}{|c|}{ - bilirubin $>42.8 \mathrm{mmol} / \mathrm{L}(>2.5 \mathrm{mg} / \mathrm{dL})$ and $/$ or PT INR $>1.5$ in combination with any elevation of AST. } \\
\hline \multicolumn{2}{|c|}{ - AST and/or ALT elevated at least five times ULN. } \\
\hline \multicolumn{2}{|c|}{ - ALP elevated at least two times ULN. } \\
\hline \multicolumn{2}{|c|}{ 2) Onset of DILI within 3 months of initiation of flucloxacillin therapy. } \\
\hline \multicolumn{2}{|c|}{ 3) Causality assessment with flucloxacillin exposure at least possible. ${ }^{b}$} \\
\hline \multicolumn{2}{|c|}{ Reference values for liver function tests } \\
\hline Laboratory test & Reference value $^{c}$ \\
\hline ALP, $\mu$ kat/L & $0.6-1.8$ \\
\hline ALT, $\mu \mathrm{kat} / \mathrm{L}$ & Men: $0.15-I . I ;$ women: $0.15-0.75$ \\
\hline AST, $\mu \mathrm{kat} / \mathrm{L}$ & Men: $0.25-0.75$; women: $0.25-0.60$ \\
\hline Bilirubin, $\mu \mathrm{mol} / \mathrm{L}$ & $5-25$ \\
\hline PT INR & $0.9-1.2$ \\
\hline
\end{tabular}

Notes: alnclusion criteria often used in studies within the DILI network. ${ }^{16-18}{ }^{6}$ Causality based on the WHO-UMC system for standardized case causality assessment. ${ }^{19}$ 'Current Swedish reference values for adults.

Abbreviations: ALP, alkaline phosphatase; ALT, alanine aminotransaminase; AST, aspartate transaminase; DILI, drug-induced liver injury; PT INR, prothrombin time international normalized ratio; ULN, upper levels of normal; UMC, Uppsala Monitoring Centre.

Table 2 Exclusion criteria

\begin{tabular}{|l|}
\hline I. Liver disease or liver injury in last 5 years \\
\hline 2. Jaundice \\
\hline 3. Hepatitis \\
\hline 4. Cirrhosis \\
\hline 5. Infection with any viral cause of hepatitis \\
\hline 6. Cancer \\
\hline 7. Gallbladder diseases \\
\hline 8. Pancreatic diseases \\
\hline 9. Heart failure \\
\hline I0. Alcohol abuse \\
\hline II. HIV infection \\
\hline 12. Rheumatoid arthritis \\
\hline 13. Sarcoidosis \\
\hline 14. Systemic lupus \\
\hline 15. Inflammatory bowel disease \\
\hline 16. Concomitant exposure to another drug more likely to cause liver \\
injury \\
\hline 17. Alcoholic hepatitis \\
\hline
\end{tabular}

Note: Cases were excluded if any of the listed conditions had been diagnosed since 1967.

level. Controls with ICD-10 codes indicating liver disease or liver ADR (Table 3) within 3 months after initiation of flucloxacillin treatment were excluded.

\section{Data analysis}

Raw data of the cases were extracted from the SWEDEGENE database and transformed into an Excel $^{\circledR}$ spreadsheet (version 14.6.8; Microsoft Corporation, Redmond, WA, USA). Desired data (demographic data, flucloxacillin data, concomitant drugs, and diagnoses) were categorized to allow statistical analyses. Drugs were coded and categorized into drug classes using the WHO Collaboration Centre for Drug Statistics Methodology International Anatomical Chemical (ATC)
Table 3 Diagnoses used for exclusion of controls

\begin{tabular}{|l|l|}
\hline ICD-I O-SE & Diagnosis \\
\hline K7I & Toxic liver injury \\
K72.0 & Acute and subacute hepatic failure \\
K76.9 & Liver disease, unspecified \\
R74.0 & Elevations of levels of ALT, AST or LDH \\
Y40 & Systemic antibiotic - adverse effect in therapeutic use \\
T88.7 & Unspecified adverse effect of drugs or medicament \\
\hline
\end{tabular}

Note: Diagnoses were collected from the Swedish National Inpatient register using ICD-IO-SE codes.

Abbreviations: ALT, alanine aminotransaminase; AST, aspartate transaminase; ICD-IO-SE, International Statistical Classification of Diseases and Related Health Problems, 10th revision, Swedish version; LDH, lactate dehydrogenase.

classification. Diagnoses and drug classes with a frequency of less than $12 \%$ among the cases were excluded for further analyses based on the statistical power calculation described further on. Liver reactions were categorized into hepatocellular, cholestatic, or mixed injury. Classification was based on the ratio $(\mathrm{R})$ between the levels of alanine aminotransaminase (ALT) and alkaline phosphatase (ALP) in multiples of their upper levels of normal (ULN), and calculated with the formula $\mathrm{R}=\left(\mathrm{ALT}_{\mathrm{ULN}} \mathrm{ULT}\right) /\left(\mathrm{ALP}_{\mathrm{ULN}} \mathrm{ULP}_{\mathrm{AP}}\right)$. The first available ALT and ALP values after the onset of DILI were used. A ratio over five counts as a hepatocellular, a ratio under two as cholestatic, and a ratio in between two and five as mixed injury. ${ }^{16-18}$

For the controls, the diagnoses present in the cases were extracted from the Swedish National IPR using ICD-10 codes. Only diagnoses documented before the collected prescription of flucloxacillin were used in comparisons. In addition, liver diagnoses within 3 months after the initiation of flucloxacillin were extracted. Drugs dispensed by a pharmacy within 3 months before and after the collection of flucloxacillin were extracted from the Swedish National 
Drug Register using ATC classifications. Sex and age at the time of collecting the flucloxacillin prescription were available in the drug excerpt. Daily dose and treatment duration of flucloxacillin were obtained from the patient instructions in the flucloxacillin prescription when available. There were no data on over-the-counter (OTC) drugs, herbal remedies or dietary supplements for the controls.

\section{Statistical analysis}

Fifty cases and 2,338 controls gave a statistical power of $\geq 80 \%$ to detect an $\mathrm{OR} \geq 4.4$ with $P<0.05$ based on a variable frequency of $\geq 12 \%$ among cases. ${ }^{22}$

JMP 12.0.1 (SAS Institute Inc., Cary, NC, USA) was used for statistical comparisons. For nominal data, number and proportion were calculated. For continuous data, median and interquartile range (IQR) were calculated. To evaluate differences between groups, Pearson's' chi-squared test was used for nominal and logistic regression for continuous data. Bonferroni correction was used to correct for multiple comparisons, giving a significance threshold of $P<0.00357$ when performing 14 independent tests $(P<0.05 / 14) . P<0.05$ indicated nominal statistical significance.

A multiple regression model was used to detect association between variables. Only variables considered reliable in the controls (eg, prescription-only drugs and diagnoses with expected high sensitivity in the Swedish national IPR), and showing significant univariate differences were included in the multiple model.

\section{Results}

In all, 652 suspected DILI cases were retrieved from the Swedish National database of spontaneously reported ADRs. Out of them, 379 cases were excluded before recruitment because of the following reasons: the ADR report was a duplicate $(n=56)$, inability to reach the reporter $(n=12)$, the physician (ie, the reporter) deemed the patient unsuitable for the study $(n=27)$, inability to reach the patient $(n=39)$, the patient chose not to participate $(n=46)$, the patient did not comply with the study procedure $(n=18)$, the patient was deceased $(n=24)$, the patient was less than 18 years old $(n=2)$, or did not fulfill study requirements or procedures $(n=155)$. The remaining 273 cases were enrolled in SWEDEGENE. Among these, 51 cases had flucloxacillin as a suspected causative drug. One case did not fulfill the DILI criteria, and was therefore excluded. In total, 50 cases of suspected flucloxacillin-induced DILI were included in this study.

Among cases, the median daily dose of flucloxacillin was $2,250 \mathrm{mg} /$ day and the median treatment duration was 10 days (Table 4). The most common indications for treatment with flucloxacillin were skin infection $(n=11)$, wound/ulcer $(n=9)$, and soft tissue infection $(n=7)$. Other indications were, eg, postoperative infection, fracture, lacrimal canaliculitis,

Table 4 Statistical comparisons between cases and controls

\begin{tabular}{|c|c|c|c|c|c|c|c|}
\hline \multirow[t]{2}{*}{ Variable } & \multicolumn{2}{|l|}{ Cases } & \multicolumn{2}{|l|}{ Controls } & \multirow[t]{2}{*}{$\mathbf{O R}^{\mathbf{a}}$} & \multirow[t]{2}{*}{$95 \% \mathrm{Cl}$} & \multirow[t]{2}{*}{$P$-value } \\
\hline & $\begin{array}{l}\text { Median } \\
\text { (IQR) }\end{array}$ & $\mathbf{n}$ & $\begin{array}{l}\text { Median } \\
\text { (IQR) }\end{array}$ & $\mathbf{n}$ & & & \\
\hline Age (years) & $63(19)$ & 49 & $67(13)$ & 2,338 & 0.91 & $0.88-0.94$ & $<0.0001$ \\
\hline Flucloxacillin dose (mg/day) & $2,250(13 \mid 2)$ & 32 & $2,250(750)$ & 2,300 & 1.00 & $1.00-1.00$ & 0.0307 \\
\hline \multirow[t]{2}{*}{ Flucloxacillin duration (days) } & $10(0)$ & 42 & $10(0)$ & 1,439 & 1.03 & $0.98-1.08$ & 0.1790 \\
\hline & n (\%) & $\mathbf{n}$ & n (\%) & $\mathbf{n}$ & OR & $95 \% \mathrm{Cl}$ & $P$-value \\
\hline Sex, female & $33(66 \%)$ & 50 & $\mathrm{I}, 084$ (46.4\%) & 2,338 & 2.25 & $1.24-4.05$ & 0.0059 \\
\hline \multicolumn{8}{|l|}{ Diagnoses (ICD- I 0-SE code) } \\
\hline Arthrosis (MI5-MI9) & $10(20.4 \%)$ & 49 & 407 (17.4\%) & 2,338 & 1.22 & $0.60-2.46$ & 0.5841 \\
\hline Hypertension (II0) & II (22.5\%) & 49 & $217(9.28)$ & 2,338 & 2.83 & $1.43-5.62$ & 0.0019 \\
\hline Kidney stones (N20-N23) & $6(12.2 \%)$ & 49 & $82(3.5 \%)$ & 2,338 & 3.84 & I.59-9.27 & 0.0013 \\
\hline \multicolumn{8}{|l|}{ Concomitant drugs (ATC code) } \\
\hline Aniline analgesics (N02BE), eg, paracetamol & $10(20.0 \%)$ & 50 & $608(26.0 \%)$ & 2,338 & 0.71 & $0.35-1.43$ & 0.3374 \\
\hline Antibacterial agents, systemic (J0I), excluding flucloxacillin & $7(14.0 \%)$ & 50 & $650(27.8 \%)$ & 2,338 & 0.42 & $0.19-0.94$ & 0.0306 \\
\hline NSAID (MOIA) & II (22.0\%) & 50 & $500(21.4 \%)$ & 2,338 & 1.04 & $0.53-2.04$ & 0.9165 \\
\hline Platelet aggregation inhibitors, excluding heparin (BOIAC) & $9(18.0 \%)$ & 50 & $523(22.4 \%)$ & 2,338 & 0.76 & $0.37-1.58$ & 0.4625 \\
\hline Sex hormones (G03) & $10(20.0 \%)$ & 50 & $234(10.0 \%)$ & 2,338 & 2.25 & I.II-4.55 & 0.0210 \\
\hline Thiazides (C03AA) & $6(12.0 \%)$ & 50 & $321(13.7 \%)$ & 2,338 & 0.86 & $0.36-2.03$ & 0.7248 \\
\hline Vitamin $\mathrm{B} I 2$ (B03BA) & 7 (I4.0\%) & 50 & I 73 (7.4\%) & 2,338 & 2.04 & $0.90-4.60$ & 0.0802 \\
\hline
\end{tabular}

Notes: ${ }^{P}$ Per change in unit. Differences were calculated with OR and Pearson's chi-squared test for categorical variables, and with logistic regression for continuous variables. Statistical significance was set to $P<0.00357$, and is shown in bold. Nominal significance was set to $P<0.05$, and is shown in bold. Patients with a liver or gallbladder condition at any time since 1967 were excluded from the study.

Abbreviations: ATC, WHO Collaboration Centre for Drug Statistics Methodology International Anatomical Chemical; ICD- I0-SE, International Statistical Classification of Diseases and Related Health Problems, I0th revision, Swedish version; IQR, interquartile range; NSAID, non-steroidal anti-inflammatory/antirheumatic drug. 
lower respiratory tract infection, and spondylodiscitis. The indication for flucloxacillin was unknown in 13 of the cases.

Seven cases $(14 \%)$ had at least one other suspected drug. They were hydrochlorothiazide $(n=2)$, amiloride $(n=1)$, candesartan $(n=1)$, clindamycin $(n=1)$, diclofenac $(n=1)$, glibenclamide $(n=1)$, interferon beta $(n=1)$, naproxen $(n=1)$, and pivmecillinam $(n=1)$. Eleven patients $(22 \%)$ had taken at least one herbal remedy and/or dietary supplement within 3 months prior to the DILI. They were calcium $(n=3)$, ginseng $(n=2)$, omega $3(n=2)$, citric acid $(n=1)$, evening primrose oil $(n=1)$, fish oil $(n=1)$, horsetail herb $(n=1)$, inositol $(n=1)$, magnesium $(n=1)$, multivitamin $(n=1)$, protein supplement $(n=1)$, rosehip ( $n=1)$, roseroot $(n=1)$, Russian root $(n=1)$, schisandra $(\mathrm{n}=1)$, silicon $(\mathrm{n}=1)$, vitamin $\mathrm{B}(\mathrm{n}=1)$, and vitamin $\mathrm{D}(\mathrm{n}=1)$. In no case was the herbal remedy and/or dietary supplement suspected by the physician to have caused the DILI.

Common clinical symptoms were icterus $(n=41)$, dark urine $(n=37)$, pale stools $(n=34)$, itch $(n=32)$, nausea $(n=32)$, abdominal pain $(n=19)$, drowsiness $(n=19)$, and fever $(n=6)$. Median maximum laboratory test values were ALP 5.9 $\mu \mathrm{kat} / \mathrm{L}$ (IQR 3.3), ALT 8.7 $\mu \mathrm{kat} / \mathrm{L}$ (IQR 7.7), aspartate transaminase (AST) $4.0 \mu \mathrm{kat} / \mathrm{L}$ (IQR 4.0), bilirubin $158.5 \mu \mathrm{mol} / \mathrm{L}$ (IQR 147.8) and prothrombin time international normalized ratio 1.0 (IQR 0.2). DILI phenotypes could be calculated for 38 of the patients and were cholestatic in $12(32 \%)$, hepatocellular in $12(32 \%)$, and mixed in 14 (37\%) of the cases. Liver biopsies were collected from 17 cases, all showing images consistent with DILI.

Among the controls, 2,343 unrelated individuals had collected at least one prescription of flucloxacillin at the pharmacy. Five controls were excluded because of liver or ADR diagnoses (Table 3 ) in the Swedish national IPR within 3 months after the collection of the prescription, which left 2,338 controls. The ICD-10 documented diagnoses were K71 toxic liver injury ( $n=2)$, R74.0 elevations of levels of ALT, AST or LDH $(n=1)$, and T88.7 unspecified adverse effect of drugs or medicament $(n=2)$.

Thirty-three (66\%) of the cases were women, compared to $1,084(46 \%)$ of the controls (Table 4). Female sex was thereby nominally, but not statistically, more common among cases than controls $(\mathrm{OR}=2.25,95 \% \mathrm{CI}=1.24-4.05, P=0.0059)$. Median age at the time of the flucloxacillin-induced DILI was 63 (IQR 19) years among the cases, while median age at the time of the first collected flucloxacillin prescription was 67 (IQR 13) years among the controls (Table 4). Age varied between 22 and 78 years among the cases, and between 47 and 97 years among the controls. Cases were significantly more likely to be younger than the controls $\left(\mathrm{OR}_{\text {per change in unit }}=0.91\right.$, 95\% CI $=0.88-0.94, P<0.0001)$. There was no difference between the groups concerning daily dose of flucloxacillin or flucloxacillin treatment duration (Table 4).

Diagnoses present in $\geq 12 \%$ of the cases and thus included in the comparisons between groups were arthrosis, hypertension, and kidney stones. Hypertension was significantly more common among cases than controls $(22.5 \%$ vs $9.3 \%$, OR $=2.83,95 \% \mathrm{CI}=1.43-5.6, P=0.0019)$, as was kidney stones (12.2\% vs 3.5\%, OR $=3.84,95 \% \mathrm{CI}=1.59-9.27, P=0.0013)$ (Table 4). For arthrosis there was no significant difference between cases and controls $(20.4 \%$ vs $17.4 \%, \mathrm{OR}=1.22$, 95\% CI $=0.60-2.46, P=0.5841)$.

Aniline analgesics, antibacterial agents excluding flucloxacillin, NSAIDs, platelet aggregation inhibitors excluding heparin, vitamin B12, and thiazides were the drug classes included for comparison between cases and controls. None of the drugs showed a statistically significant difference between the groups (Table 4). Sex hormones were nominally significantly more common among cases than controls ( $20 \%$ vs $10 \%$, OR $=2.25,95 \% \mathrm{CI}=1.11-4.55, P=0.0210)$, but this significance disappeared when adjusting for sex, ie, the effect of female sex seemed to be responsible for the sex hormone effect. Furthermore, there was no significant difference between female cases and controls regarding intake of sex hormones $(30.3 \%$ vs $21.6 \%$, OR $=1.58,95 \%$ $\mathrm{CI}=0.74-3.36, P=0.2326)$. Systemic antibacterial agents excluding flucloxacillin were nominally significantly more uncommon among cases than controls (14\% vs $27.8 \%$, OR $=0.42,95 \% \mathrm{CI}=0.19-0.94, P=0.0306$ ).

Sex, kidney stones, and antibacterial agents excluding flucloxacillin were tested for their contribution in a multiple regression model (Table 5). In this model female sex and kidney stones showed a significant difference between the groups. Treatment with an antibacterial agent excluding flucloxacillin showed a nominally significant difference between the groups.

\section{Discussion}

The main results of this study were that female sex, a diagnosis of hypertension, and kidney stones were more common among cases than controls. Female sex as a risk factor for fluclox-

Table 5 Multiple regression model comparing cases and controls

\begin{tabular}{|l|l|l|l|}
\hline Variable & OR & $\mathbf{9 5 \%} \mathbf{C l}$ & P-value \\
\hline Antibacterial agents & \\
Kidney stones & 0.38 & $0.17-0.85$ & $\mathbf{0 . 0 1 8 8}$ \\
Sex, female & $5.5 \mathrm{I}$ & $2.2 \mathrm{I}-13.72$ & $\mathbf{0 . 0 0 0 3}$ \\
\hline
\end{tabular}

Notes: ${ }^{a}$ Excluding flucloxacillin. Only variables considered reliable were included in the analysis. Statistical significance was set to $P<0.00357$, and is shown in bold. Nominal significance was set to $P<0.05$, and is shown in bold. 
acillin-induced DILI is consistent with previous studies. ${ }^{1}$ In relation to this, it is interesting that the HLA-B*57:01 genotype tends to be more common among women than men who suffer from flucloxacillin-induced DILI. ${ }^{12}$ To our knowledge, previous studies have not shown any diagnosis or concomitant drug as a risk factor for flucloxacillin-induced DILI. In this study, hypertension and kidney stones were statistically more common among cases than controls. The diagnosis of hypertension is expected to have a low sensitivity among controls, since the Swedish IPR has incomplete coverage of diagnoses from primary care..$^{21}$ The Swedish IPR has $99 \%$ coverage of all inpatient hospital diagnoses, and approximately $80 \%$ coverage of hospital-based outpatient care diagnoses. Sensitivity is estimated to vary between diagnoses; from $>90 \%$ for a typical inpatient diagnosis such as myocardial infarction to $\sim 10 \%$ for a typical outpatient diagnosis such as hypertension. ${ }^{21}$ Due to the low sensitivity of this diagnosis, we believe that the finding of hypertension as a potential risk factor is the result of bias. A diagnosis of kidney stones should be more reliable, since this is often diagnosed at a hospital. In the univariate analysis, a statistically significant, almost 4-fold increased risk for flucloxacillin-induced DILI was shown in individuals with a medical history of kidney stones, and in the multiple regression model this risk was 5-fold. Non-alcoholic liver disease and gallstone disease have previously been shown to be associated with kidney stones. ${ }^{23-25}$ The mechanisms linking these diseases with kidney stones are complex and not yet established. In our study, patients with preexisting liver and gall bladder diseases where excluded, and it cannot be ruled out that persisting changes in urinary constituents predispose to liver reactions.

This study also found a statistically significant difference between cases and controls regarding age, suggesting young age as a risk factor. This is opposed to previous studies that have shown age older than 60 years as a risk factor. ${ }^{1}$ This reverse result could be due to the fact that controls in the TWINGENE database were born 1911-1958, and the Swedish National Drug Register was established in 2005. ${ }^{15,26}$ Thus, the controls were at least 47 years old when their first prescription was documented.

\section{Strength and limitations}

The study has some limitations. 1) Selection bias cannot be excluded since there is a risk that older and unhealthier individuals do not participate in studies. 2) There is a risk of unreliable or incomplete information in interviews, medical records or the Swedish IPR. 3) The controls' intake of OTC drugs, herbal remedies, and dietary supplements was not captured and could therefore not be compared with the cases.
However, the herbal remedies and dietary supplements taken by the cases are not known to cause liver damage in normal doses. ${ }^{27}$ 4) Finally, some cases had other suspected causative drugs in addition to flucloxacillin.

Strengths of this study are the well-established method of patient recruitment and data collection, the well-defined cases, and that Sweden's national registers and records were used for characterization of the controls.

\section{Conclusion}

The strongest known risk factor for DILI associated with flucloxacillin is HLA-B*57:01. ${ }^{3}$ New and rapid methods for HLA testing are needed to make preemptive testing clinically feasible, and due to the low prevalence of this type of DILI $(\sim 1$ in 10,000$)$, thousands of patients would need to be tested to avoid one case. ${ }^{13}$ HLA-B*57:01 is at present more useful as part of diagnostics in patients with evidence of a liver reaction. ${ }^{28}$ HLA typing of all patients starting flucloxacillin is not cost-effective, but clinical risk factors for DILI could be used to indicate whom to test. We established female sex as a risk factor for flucloxacillin-induced DILI, and a history of kidney stones was identified as a potential risk factor. Potentially females with a history of kidney stones should be tested for the genetic risk factor HLA-B*57:01, but this needs to be confirmed in further studies.

\section{Acknowledgments}

We thank research nurses Ulrica Ramqvist, Elisabeth Stjernberg, Charlotta Haglund, and Elisabeth Balcom, and research assistants Sofie Collin, Eva Prado Lopez, Agnes Kataja Knight, Agnes Wadelius, and Martha Wadelius, Department of Medical Sciences, Clinical Pharmacology, Uppsala University, Uppsala, Sweden, for recruiting and interviewing cases and for administering the phenotype database. We are grateful for statistical advice from Niclas Eriksson at Uppsala Clinical research Center - UCR, Uppsala, Sweden, and indebted to Patrik Magnusson and Barbro Sandin at the Swedish Twin Registry, Department of Medical Epidemiology and Biostatistics, Karolinska Institutet, Stockholm, Sweden for providing clinical data for the controls. The Swedish Twin Registry is managed by Karolinska Institutet and receives funding through the Swedish Research Council under the grant number 2017-00641.

This work was supported by the Swedish Research Council (Medicine 521-2011-2440 and 521-2014-3370); Swedish Heart and Lung Foundation (20120557, 20140291 and 20170711); Selander's Foundation; Thuréus' Foundation; the Swedish Medical Products Agency; and the Clinical Research Support (Avtal mellan svenska staten 
och vissa landsting om samarbete om grundutbildning av läkare, medicinsk forskning och utveckling av hälso och sjukvården (ALF) [Agreement between the Swedish State and counties on cooperation on undergraduate medical education, medical research and development of health care]) at Uppsala University.

\section{Disclosure}

The authors report no conflicts of interest in this work.

\section{References}

1. Russmann S, Kaye JA, Jick SS, Jick H. Risk of cholestatic liver disease associated with flucloxacillin and flucloxacillin prescribing habits in the UK: cohort study using data from the UK General Practice Research Database. Br J Clin Pharmacol. 2005;60(1):76-82.

2. Fairley CK, Mcneil JJ, Desmond P, et al. Risk factors for development of flucloxacillin associated jaundice. BMJ. 1993;306(6872):233-235.

3. Fontana RJ. Pathogenesis of idiosyncratic drug-induced liver injury and clinical perspectives. Gastroenterology. 2014;146(4):914-928.

4. Pirmohamed M. Pharmacogenetics of Idiosyncratic Adverse Drug Reactions. In: Uetrecht J, editor. Adverse Drug Reactions. Handbook of Experimental Pharmacology. Vol 196. Berlin, Heidelberg: Springer; 2010;477-491.

5. Yip VL, Alfirevic A, Pirmohamed M. Genetics of immune-mediated adverse drug reactions: a comprehensive and clinical review. Clin Rev Allergy Immunol. 2015;48(2-3):165-175.

6. Naisbitt DJ, Pirmohamed M, Park BK. Immunopharmacology of hypersensitivity reactions to drugs. Curr Allergy Asthma Rep. 2003;3(1):22-29.

7. Pirmohamed M, Park BK. Adverse drug reactions: back to the future. Br J Clin Pharmacol. 2003;55(5):486-492.

8. Björnsson ES. Epidemiology and risk factors for idiosyncratic druginduced liver injury. Semin Liver Dis. 2014;34(2):115-122.

9. Takai S, Higuchi S, Yano A, et al. Involvement of immune- and inflammatory-related factors in flucloxacillin-induced liver injury in mice. J Appl Toxicol. 2015;35(2):142-151.

10. Monshi MM, Faulkner L, Gibson A, et al. Human leukocyte antigen (HLA)-B*57:01-restricted activation of drug-specific T cells provides the immunological basis for flucloxacillin-induced liver injury. Hepatology. 2013;57(2):727-739.

11. Lakehal F, Dansette PM, Becquemont L, et al. Indirect cytotoxicity of flucloxacillin toward human biliary epithelium via metabolite formation in hepatocytes. Chem Res Toxicol. 2001;14(6):694-701.
12. Daly AK, Donaldson PT, Bhatnagar P, et al. HLA-B*5701 genotype is a major determinant of drug-induced liver injury due to flucloxacillin. Nat Genet. 2009;41(7):816-819.

13. Phillips EJ, Mallal SA. HLA-B*5701 and flucloxacillin associated drug-induced liver disease. AIDS. 2013;27(3):491-492.

14. Russmann S, Jetter A, Kullak-Ublick GA. Pharmacogenetics of druginduced liver injury. Hepatology. 2010;52(2):748-761.

15. Magnusson PK, Almqvist C, Rahman I, et al. The Swedish Twin Registry: establishment of a biobank and other recent developments. Twin Res Hum Genet. 2013;16(1):317-329.

16. Chalasani N, Fontana RJ, Bonkovsky HL, et al. Causes, clinical features, and outcomes from a prospective study of drug-induced liver injury in the United States. Gastroenterology. 2008;135(6):1924-1934.

17. Chalasani N, Bonkovsky HL, Fontana R, et al. Features and Outcomes of 899 Patients With Drug-Induced Liver Injury: The DILIN Prospective Study. Gastroenterology. 2015;148(7):1340-1352.

18. Fontana RJ, Watkins PB, Bonkovsky HL, et al. Drug-Induced Liver Injury Network (DILIN) Prospective Study. Drug Saf. 2009;32(1):55-68.

19. The use of the WHO-UMC system for standardised case causality assessment. Available from: http://www.who.int/medicines/areas/quality_safety/safety_efficacy/WHOcausality_assessment.pdf. Accessed November 6, 2018.

20. Socialstyrelsen.se. Diagnoskoder (ICD-10). Available from: http:// www.socialstyrelsen.se/klassificeringochkoder/diagnoskodericd-10. Accessed October 23, 2018.

21. Ludvigsson JF, Andersson E, Ekbom A, et al. External review and validation of the Swedish national inpatient register. BMC Public Health. 2011;11:450.

22. Dean AG, Sullivan KM, Soe MM. Open Source Epidemiologic Statistics for Public Health, Version 3.01. Available from: http://www.openepi. com. Accessed 3 May 2017.

23. Nam IC. Association of non-alcoholic fatty liver disease with renal stone disease detected on computed tomography. Eur J Radiol Open. 2016;3:195-199.

24. Einollahi B, Naghii MR, Sepandi M. Association of nonalcoholic fatty liver disease (NAFLD) with urolithiasis. Endocr Regul. 2013;47(1):27-32.

25. Ahmed MH, Barakat S, Almobarak AO. The association between renal stone disease and cholesterol gallstones: the easy to believe and not hard to retrieve theory of the metabolic syndrome. Ren Fail. 2014;36(6):957-962.

26. socialstyrelsen.se. Läkemedelsregistret [Swedish National Drug Register]. Available from: http://www.socialstyrelsen.se/register/halsodataregister/lakemedelsregistret. Accessed February 27, 2017.

27. The LiverTox database. Available from: https://livertox.nlm.nih.gov/. Accessed 2017 Dec 28.

28. Alfirevic A, Pirmohamed M. Genomics of Adverse Drug Reactions. Trends Pharmacol Sci. 2017;38(1):100-109.
Drug, Healthcare and Patient Safety

\section{Publish your work in this journal}

Drug, Healthcare and Patient Safety is an international, peer-reviewed open access journal exploring patient safety issues in the healthcare continuum from diagnostic and screening interventions through to treatment, drug therapy and surgery. The journal is characterized by the rapid reporting of reviews, original research, clinical, epidemiological and

\section{Dovepress}

post-marketing surveillance studies, risk management, health literacy and educational programs across all areas of healthcare delivery. The manuscript management system is completely online and includes a very quick and fair peer-review system. Visit http://www.dovepress.com/ testimonials.php to read real quotes from published authors. 